\title{
Directivity and Bandwidth Enhancement of Patch Antenna using Metamaterial
}

\author{
Ranjeet Pratap Singh Bhadoriya \\ ${ }^{I}$ Professor Electronics \& Communication Department, IPS College of Technology \& Management, Gwalior, India \\ *Correspondence: Ranjeet Pratap Singh Bhadoriya; Email: r.pratap7872@gmail.com
}

\begin{abstract}
This manuscript is the outcome of detailed research. A novel metamaterial structure is proposed in this paper to improve the directivity of the antenna. In this research paper a method of implementing metamaterial over the patch is used to enhance the directivity of rectangular microstrip patch antenna. From the results proposed expectation has been achieved, it is noted that in the presence of the LHM, the antenna is more directive and has a higher gain. This proposed patch is designed at the frequency of $2.75 \mathrm{GHz}$. The proposed structure is a combination of circular rings by virtue of its backward wave propagation property and negative reflection it improve the parameters of antenna.
\end{abstract}

Keywords: Negative Media, RMPA, return loss, multiband, directivity.

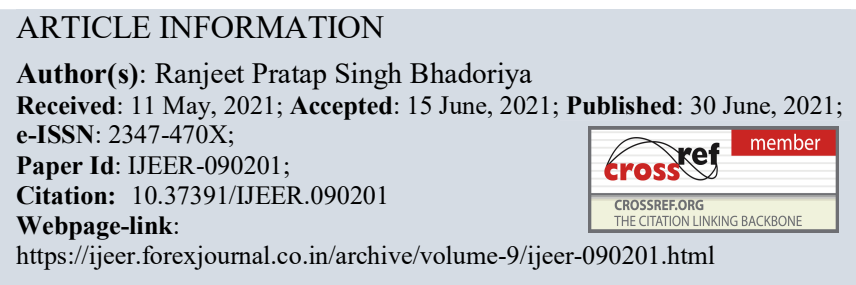

\section{INTRODUCTION}

Nowadays, the term metamaterials can be found frequently in the literature. The prefix "meta" in Greek means "beyond". According to a general definition, metamaterials are usually used to refer the artificial material which has some electromagnetic properties which are not common in the nature [6]. In nature the permittivity and the permeability of most materials are positive. The material with positive permittivity and permeability are referred as right-handed material (RHM). The medium with simultaneously negative values of permittivity $\varepsilon$ and permeability $\mu$ was initially proposed by Veselago [2]. G. V. Eleftheriades [12] presented clear experimental evidence confirming negative refraction and went even further to demonstrate for the first time focusing of electromagnetic waves from a left-handed lens. The structure used was a $2 \mathrm{D}$ periodically L-C loaded TL network (dual TL medium). Claudio G. Parazzoli [13] at Boeing Phantom Works at Seattle carried out an experiment similar to that made by Smith in [5]. Using a free-space measurement setup, they detected negatively refracted waves at a remarkably long distance from the LHM sample, thereby dispelling any doubt concerning the far field nature of these waves. These results, which fully supported the theoretical results of $[16,17]$, clearly confirmed the existence of negative refraction. G. V. Eleftheriades [15] presented simulation results showing sub wavelength focusing capability of a LHM lens. They used a 2D dual TL medium sandwiched between two 2D conventional TL media (right-handed media). Evidence of growing evanescent waves within the dual TL medium was shown for both infinite and finite length structures. In December 2003, they published further analytical and simulation results on sub wavelength focusing. In particular, they discussed the required criteria for perfect focusing, as well as the restrictions imposed on the resolution by the periodicity of the LHM used.

\section{Calculation}

CST-MSW was used for designing and simulation of the proposed microstrip antenna alone and with metamaterial cover. Dimension of patch antenna was calculated for the operating frequency of $2.75 \mathrm{GHz}$. Substrate used was FR4 lossy which has dielectric constant of 4.3 and height $1.6 \mathrm{~mm}$.

After calculation of dimensions using formulas stated in [1], Length and width of the proposed RMPA were calculated by using formulas in [1], and the designed RMPA is shown in figure 1. The simulated results of the proposed RMPA are shown in subsequent figures from 2 to 4 .

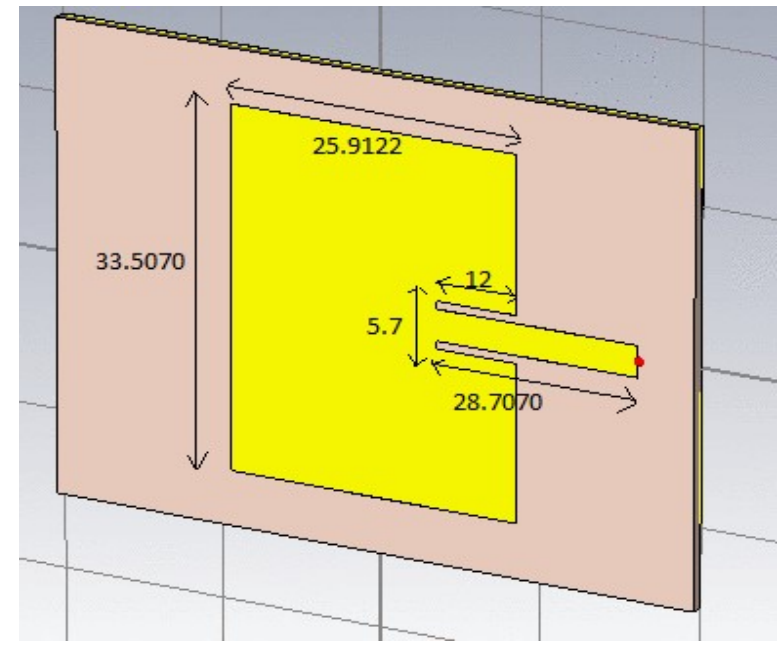

Figure 1: RMPA at $2.75 \mathrm{GHz}$. 


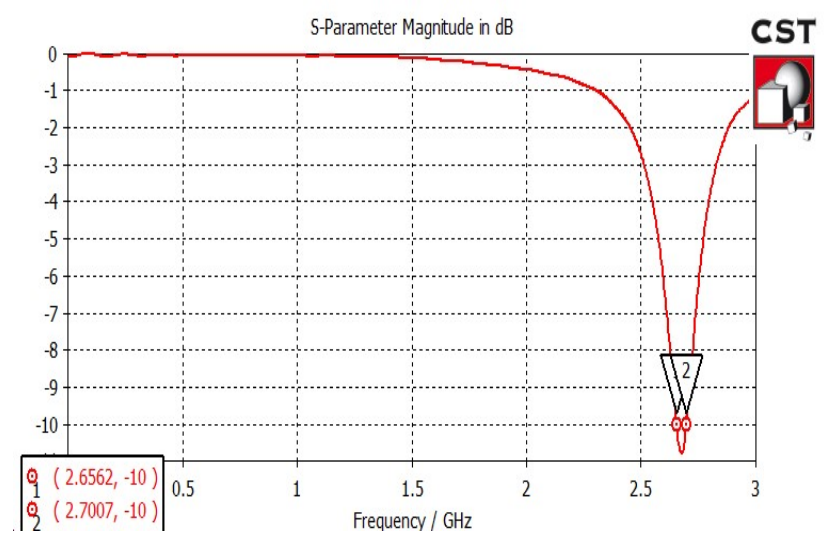

Figure 2: Simulated result of RMPA shown in Fig. 1

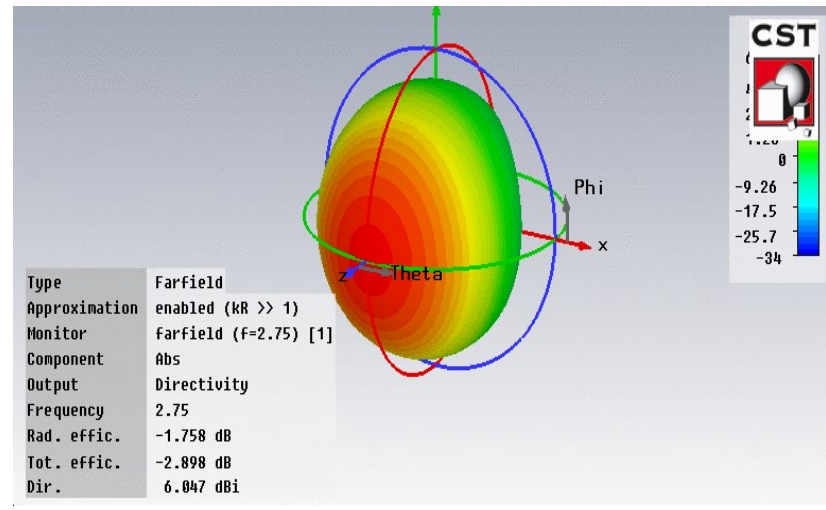

Figure 3: Simulation result shows directivity, efficiency and radiation pattern.

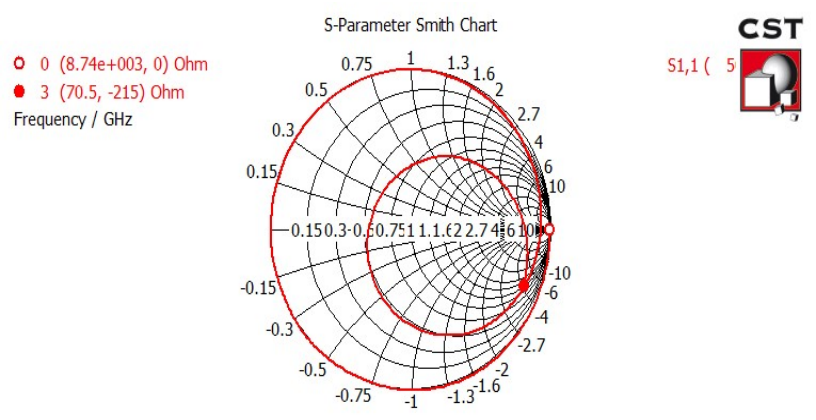

Figure 4: Smith Chart of the proposed patch without metamaterial introduction.

After simulation it appeared that the proposed antenna parameters are not fulfilling the requirement so a metamaterial structure is incorporated over this proposed patch. This metamaterial implementation modifies the antenna parameters to a great extent. e. g. return loss [6], bandwidth after implementation of metamaterial also increased than the bandwidth of RMPA alone. All the results after implementation of metamaterial are shown in fig. 6 to 8 . And metamaterial structure shown in fig. 5.

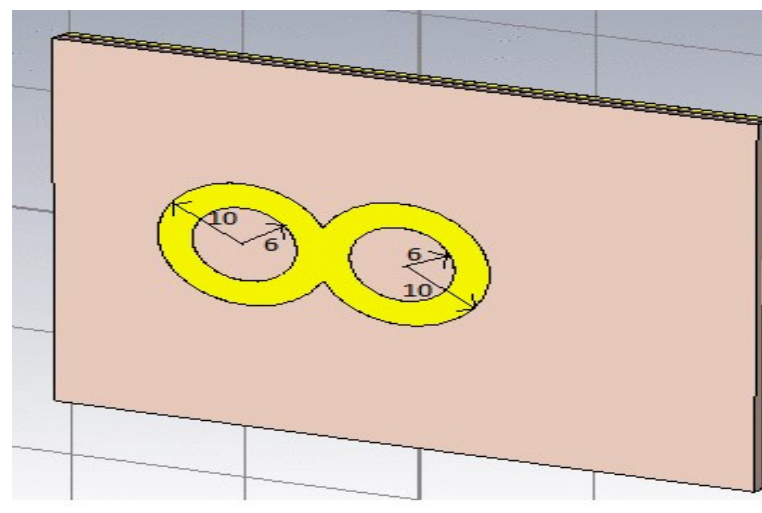

Figure 5: Proposed LH material design at the height of $3.276 \mathrm{~mm}$ from base.

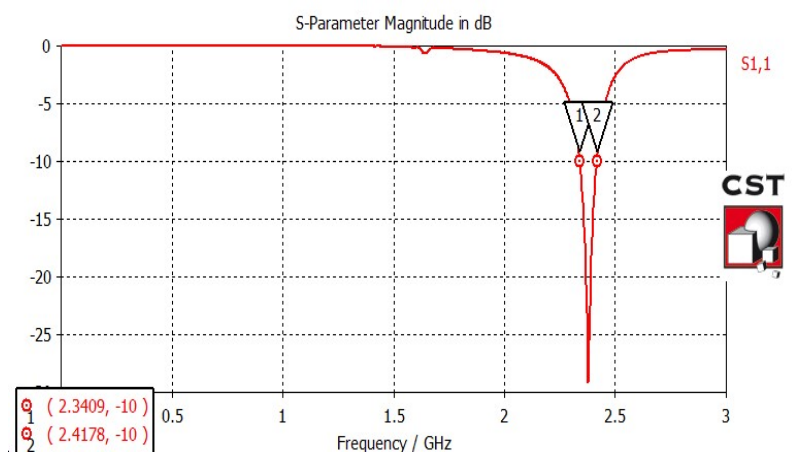

Figure 6: This is the simulated result of design in figure 4, dip at $2.37 \mathrm{GHz}$. The value of return loss and bandwidth was introduced before.

After introduction of metamaterial the parameters of patch modifies drastically, return loss has been increased from $10.5 \mathrm{~dB}$ to $-29 \mathrm{~dB}$ and bandwidth also increased from $44 \mathrm{GHz}$ to $77 \mathrm{GHz}$. Radiation pattern result are listed follows.

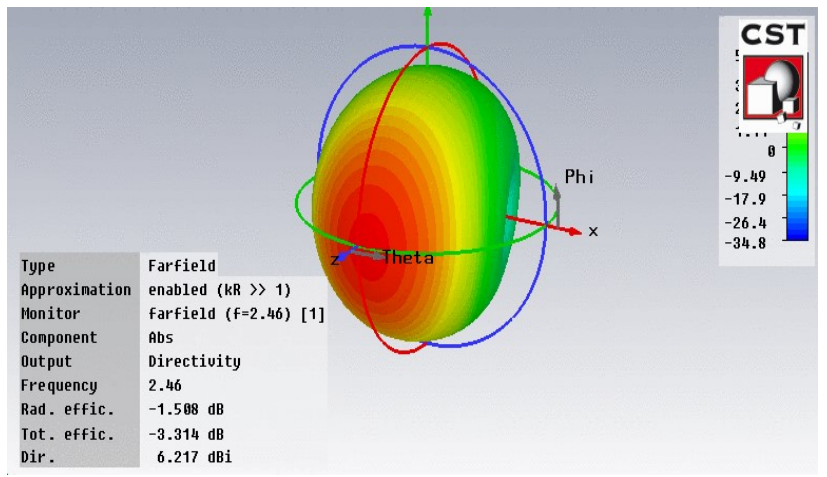

Figure 7: This is the simulated result of design in figure 4, showing radiation pattern with efficiency and directivity. 


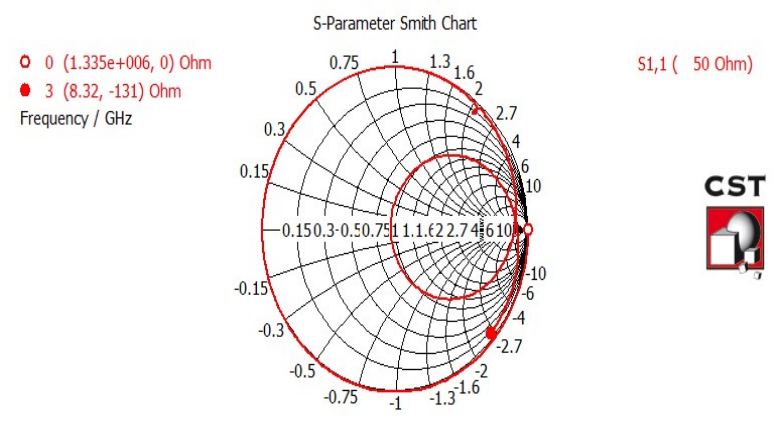

Figure 8: Smith Chart of the proposed patch after metamaterial introduction.

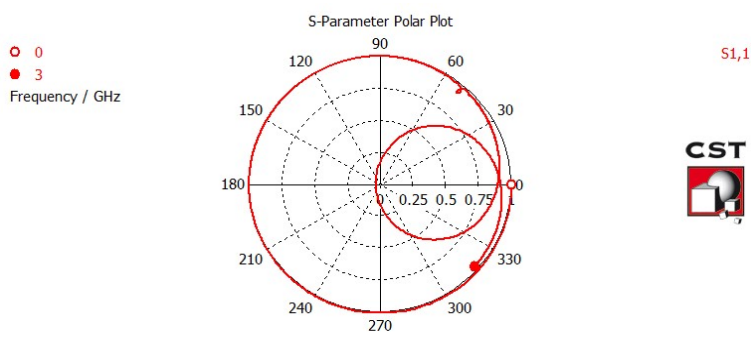

Figure 9: Polar plot of the proposed patch after metamaterial introduction.

\section{RESULT}

After simulation of RMPA alone and after metamatarial it has been observed that the antenna performance parameters have been increased drastically after introducing metamaterial cover. These results are compared w.r.t the parameter variation. Comparative chart is shown below in table 1.

TABLE I: COMPARISON CHART

\begin{tabular}{|l|l|l|l|}
\hline S. no. & Parameters & $\begin{array}{l}\text { Parameters of } \\
\text { RMPA alone at } \\
\mathbf{2 . 7 5} \mathbf{~ G H z}\end{array}$ & $\begin{array}{l}\text { After metamaterial } \\
\text { introduction } \\
\text { at } 2.45 \mathbf{~ G H z}\end{array}$ \\
\hline 1 & Return loss & $-10.5 \mathrm{~dB}$ & $-29 \mathrm{~dB}$ \\
\hline 2 & Bandwidth & $44 \mathrm{MHz}$ & $77 \mathrm{MHz}$ \\
\hline 3 & Directivity & $6.047 \mathrm{~dB}$ & $6.217 \mathrm{~dB}$ \\
\hline 4 & Efficiency & $76 \%$ & $77 \%$ \\
\hline
\end{tabular}

After the comparison it has been observed that the proposed metamterial structure converted the single band antenna to double band without affecting its parameters and bandwidth has also increased as desired.

\section{औ 4. CONCLUSION AND FUTURE SCOPE}

The designed antenna could be used in wireless communication for S band. Antenna will be able to propagate at frequency of 2.45 by applying a resonant frequency of 2.75 $\mathrm{GHz}$. Return losses of the propagating frequencies increased from $-10.5 \mathrm{~dB}$ to $-29 \mathrm{~dB}$ as well as Bandwidth of the antenna have drastically improved from $44 \mathrm{MHz}$ to $77 \mathrm{MHz}$ after introduction of metamaterial cover. This proposed design by authors can reduce the size of antenna required because frequency has been shifted from 2.75 to $2.45 \mathrm{GHz}$, this theory was proposed in [8]. This proposed design could be useful when more directive antenna is required.

\section{Z REFERENCES}

Book References

[1] C. A. Balanis, "Microstrip Antenna" in Antenna Theory and Design, Vol 3, John Wiley \& Sons, Inc., 1997, pp. 811-882.

[2] D. M. Pozar, "Introduction to microwave system" in Microwave Engineering, 4th Edition. John Wiley \& Sons 2004, pp. 658-99.

Journal References

[3] V. G. Veselago, "The electrodynamics of substances with simultaneously negative value $\varepsilon$ and $\mu$ ", Sov. Phys. Uspekekh, Vol no. 10 (4), pp. 509-514, 1968

[4] J. B. Pendry, "Negative refraction males a prefect lens", Phys Rev Lett, 85, pp. 3966-3969, 2000.

[5] R. A. Shelby, D. R. Smith, S. Shultz, "Experimental Verification of a Negative Index of Refraction," Science Vol. 292, pp. 77-79, 2001.

[6] N. Garcia and M. Nieto-Vesperinas, "Nieto-Vesperinas and Garcia Reply," Physical Review Letters, vol. 91, no. 9, p. 099702, 2003.

[7] P. K. Singhal et al., "Design and Characterization of Compact Microstrip Patch Antenna Using "Split Ring" Shaped Metamaterial Structure" IJECE, Vol.2, No.5, pp. 655 662, 2012

[8] R Bhadoriya et al, "Miniaturisation of WLAN feeler using media with a negative refractive index". BIJIT, Vol.5, No.1, 2013.

[9] Ahmad A. Sulaiman et al., "Bandwidth Enhancement in patch antenna by metamaterial substrate", European Journal of scientific research, Vol. 44 No. 3, pp. 493-501, 2010.

[10] Huda A. Mazid, Mohammad Kamal A. Rahim, Thelasa Masri, "Left-handed metamaterial design for microstrip antenna application", IEEE International, RF and Microwave conference, pp. 218-221, 2008.

[11] H. A. Majid, M. K. A. Rahim and T. Marsi, "Microstrip Antenna gain enhancement using left-handed metamaterial structure", progress in Electromagnetic Research M. Vol.8, 235-247, 2009.

[12] G. V. Eleftheriades, A. K. Iyer, and P. C. Kremer, "Planar Negative Refractive Index Media Using Periodically L-C Loaded Transmission Lines," in IEEE Transactions on 
Microwave Theory and Techniques, vol. 50, no. 12, pp. 27022712, 2002.

[13] C. G. Parazzoli, R. B. Greegor, K. Li, B. E. C. Koltenbah, and M. Tanielian, "Experimental Verification and Simulation of Negative Index of Refraction using Snell's Law," Physical Review Letters, vol. 90, no. 10, p. 107401, 2003.

[14] R. P. Singh Bhadoriya and S. Nigam, "Bandwidth enhancement and modification of single band patch antenna into double band," 2016 3rd International Conference on Computing for Sustainable Global Development (INDIACom), 2016, pp. 10291032.

[15] A. Grbic and G. V. Eleftheriades, "Growing Evanescent Waves in Negative-Refractive-Index Transmission-Line Media," Applied Physics Letters, vol. 82, no. 12, pp. 1815-1817, 2003.

[16] D. R. Smith, D. Schurig, and J. B. Pendry, "Negative Refraction of Modulated Electromagnetic Waves," Applied Physics Letters, vol. 81, no. 15, pp. 2713-2715, 2002.

[17] J. Pacheco, T. M. Grzegorczyk, B.-I. Wu, Y. Zhang, and J. A. Kong, "Power Propagation in Homogeneous Isotropic Frequency-Dispersive Left-Handed Media," Physical Review Letters, vol. 89, no. 25, p. 257401, 2002.

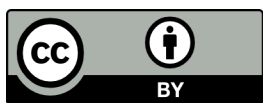

(C) 2021 by the Ranjeet Pratap Singh Bhadoriya. Submitted for possible open access publication under the terms and conditions of the Creative Commons Attribution (CC BY) license (http://creativecommons.org/licenses/by/4.0/). 